\title{
AVALIAÇÃO DA FREQUÊNCIA EM CONSULTAS NUTRICIONAIS DOS PACIENTES APÓS CIRURGIA BARIÁTRICA
}

\author{
Nutritional consultations frequency after bariatric surgery
}

\author{
Ana Luiza Savaris MENEGOTTO, Magda Rosa Ramos CRUZ, Fernando Lucas SOARES, \\ Mario Gilberto Jesus NUNES, Alcides José BRANCO-FILHO
}

Trabalho realizado no Centro Avançado de Videolaparoscopia do Paraná (CEVIP), Curitiba, Paraná, Brasil.

DESCRITORES - Obesidade mórbida Cirurgia bariátrica. Período pós-operatório.
RESUMO - Racional - Com o crescimento das intervenções cirúrgicas para reduzir a obesidade, há necessidade de acompanhamento nutricional periódico a longo prazo para garantir o estado nutricional dos pacientes. Objetivo - Avaliar a adesão ao acompanhamento nutricional periódico dos pacientes submetidos à cirurgia bariátrica. Métodos - Foram coletados dados das fichas de cadastro do serviço de nutrição de pacientes que realizaram operação bariátrica entre 2001 e 2008. Resultados - Foram avaliadas 469 fichas. Delas 83\% eram de mulheres e 16,8\% de homens com média de idade de 38,2 $\pm 12,03$ anos. O acompanhamento apresentou média de 2,04 \pm 0,77 consultas no pré-operatório e 3,4 \pm 9,19 consultas no pósoperatório e houve redução progressiva da assiduidade destes pacientes em relação ao tempo pós-cirúrgico. Conclusão - Verificou-se alta evasão no acompanhamento nutricional no pós-operatório, sendo preocupante a alta desistência principalmente após dois anos, o que pode resultar em complicações metabólicas e recuperação do peso perdido.

\section{Correspondência:}

Ana Luiza Savaris Menegotto

e-mail: savaris_ana@yahoo.com.br

Fonte de financiamento: não há

Conflito de interesses: não há

Recebido para publicação: 27/09/2012

Aceito para publicação: 11/12/2012

HEADINGS - Obesity, morbid. Bariatric surgery. Postoperative Period.
ABSTRACT - Background - With the growth of surgical interventions to reduce obesity, there is a necessity of a periodic nutritional attendance at long term to ensure the nutritional status of the patients. Aim - To assess the adherence to the periodic nutritional attendance of patients undergoing bariatric surgery. Methods - Data were collected from registration forms of a nutrition service from patients who underwent bariatric surgery between 2001 and 2008. Results - Were evaluated 469 registration forms, of which $83 \%$ corresponded to female and $16.8 \%$ to male, with a mean age of $38.2 \pm 12.03$ years. The attendance showed an average of 2.04 \pm 0.77 pre-operatively queries and $3.4 \pm 9.19$ post-operative queries and there was progressive reduction in assiduity of these patients in surgical follow-up. Conclusion - There was a high dropout of the patients in post-operative nutritional attendance, being primarily concerned about the high dropout after two years of surgery; this tendency may result in metabolic complications and regain of lost weight.

\section{INTRODUÇÃO}

$\mathrm{O}$ significativo aumento mundial dos casos de obesidade mórbida vem resultando no crescimento das intervenções cirúrgicas para correção deste mal. Intenciona reduzir, através da perda significativa do excesso de peso, o risco de mortalidade e comorbidades hipertensão arterial sistêmica, diabete melito tipo 2, apnéia obstrutiva do sono, dislipidemia e doença hepática ${ }^{19}$. Contudo, a indicação do tratamento cirúrgico deve ser sugerida através de avaliação clínica realizada por equipe multidisciplinar ${ }^{20}$, considerando o histórico de tratamentos pregressos não invasivos para perda de peso como utilização de medicamentos, acompanhamento nutricional e prática de atividades físicas ${ }^{10}$.

No Brasil, a pesquisa entre os anos de 2008 e 2009 de Orçamentos Familiares do IBGE demonstrou prevalência de obesidade na população brasileira de $16,9 \%$ e $12,4 \%$ entre as mulheres e os homens respectivamente ${ }^{12}$. 
Segundo o Ministério da Saúde, foram realizadas em 2008 um total de 3.195 operações bariátricas em hospitais que prestam serviços ao Sistema Único de Saúde (SUS), mostrando aumento significativo de 542\% desde o ano de $2001^{16}$.

Entretanto, para resultados efetivos no pósoperatório, o acompanhamento nutricional deve ser periódico e a longo prazo, garantindo alimentação adequada tanto em quantidade como em qualidade. Com o decorrer do tempo, podem surgir casos de carências nutricionais, devido à ingestão alimentar deficiente e a síndrome de má-absorção de vitaminas e minerais, resultando em desnutrição, neuropatias, intolerâncias alimentares e outras enfermidades ${ }^{1,9,14,21}$.

Visando verificar o comprometimento do paciente, sua alimentação e manutenção de peso, este estudo tem como objetivo avaliar a adesão ao acompanhamento nutricional periódico dos pacientes submetidos à cirurgia bariátrica.

\section{MÉTODOS}

Estudo retrospectivo observacional foi organizado para coletar dados das fichas de cadastro do Serviço de Nutrição do Centro Avançado de Videolaparoscopia do Paraná (CEVIP), Curitiba, Paraná, Brasil, de pacientes atendidos entre os anos de 2001 a 2010, transcritos e analisados através do software Microsoft Excel ${ }^{\circledR}$. Foram selecionados pacientes que realizaram operação bariátrica (bypass em Y-de-Roux ou derivação duodenal) entre 2001 e 2008. Pacientes que realizaram mais de uma operação ou outras técnicas cirúrgicas foram excluídos do estudo.

Todos os pacientes realizaram no mínimo duas consultas no pré-operatório, tendo seus retornos no pós-operatório pré-determinados, ambos conforme protocolo do serviço. Os retornos às consultas de nutrição foram classificados em relação ao tempo em dias após a operação: $1^{\circ}$ mês ( 0 a 30 dias), $2^{\circ}$ ao $3^{\circ}$ mês ( 31 a 90 dias), $2^{\circ}$ trimestre (91 a 180 dias), $3^{\circ}$ trimestre (181 a 270 dias), $4^{\circ}$ trimestre a 2 anos ( 271 a 720 dias), acima de dois anos (acima de 721 dias).

As variáveis foram analisadas descritivamente através de distribuição de frequência percentual, média e desvio-padrão.

\section{RESULTADOS}

Foram avaliados 469 fichas das quais 83\% correspondiam a mulheres e $16,8 \%$ a homens. A média de idade foi de 38,2 $\pm 12,03$ anos.

O acompanhamento apresentou média de 2,04 $\pm 0,77$ consultas no pré-operatório e de 3,4 \pm 9,19 - variando de 0 a 20 consultas - no pós-operatório. Verificou-se redução progressiva da assiduidade destes pacientes em relação ao tempo pós-cirúrgico (Tabela 1).
TABELA 1 - Assiduidade dos pacientes para consulta após cirurgia bariátrica

\begin{tabular}{|l|c|c|}
\hline & Prevalência & Número médio de consultas \\
\hline $1^{\circ}$ mês & $81,45 \%$ & $1,22 \pm 0,08$ \\
\hline $2^{\circ}$ ao $3^{\circ}$ mês & $51,81 \%$ & $0,66 \pm 0,78$ \\
\hline $2^{\circ}$ trimestre & $33,90 \%$ & $0,40 \pm 0,62$ \\
\hline $3^{\circ}$ trimestre & $25,59 \%$ & $0,33 \pm 0.67$ \\
\hline $4^{\circ}$ trimestre a dois anos & $25,37 \%$ & $0,50 \pm 1,31$ \\
\hline Acima de dois anos & $14,93 \%$ & $0,31 \pm 1$ \\
& & DISCUSSA O
\end{tabular}

O predomínio de mulheres na amostra provavelmente decorre do fato destas procurarem mais o tratamento para o controle da obesidade ${ }^{10,11,12,13}$.

A média da idade encontrada na população estudada mostra valor semelhante a outros estudos realizados com obesos mórbidos, evidenciando população relativamente jovem já necessitando de um procedimento tão complexo para o tratamento da obesidade ${ }^{2,4,15}$.

Foi observada redução progressiva da assiduidade dos pacientes em relação ao tempo pós-operatório, ao contrário do publicado por Alvarado et al. ${ }^{2}$, onde $87,8 \%$ dos pacientes avaliados aderiram ao acompanhamento no pós-operatório pelo período mínimo de um ano.

$A$ adesão às consultas foi maior no primeiro trimestre, decaindo significativamente até o primeiro ano de pós-operatório. Tal fato denota a maior preocupação dos pacientes no período inicial após o ato cirúrgico, onde a dieta possui características específicas e a as complicações são maiores; todavia, reflete a não compreensão do principal objetivo do procedimento que é auxiliar o processo de reeducação alimentar, evitando deficiências nutricionais e perda de peso inadequada.

No que diz respeito aos retornos para consulta após dois anos, observou-se grande redução - cerca de $85 \%$ - na frequência dos pacientes. Avaliando pósoperatório com mais de cinco anos, Hernández et al. ${ }^{11}$ demostraram que $81,5 \%$ dos pacientes desistiram do acompanhamento, ou seja, resultado semelhante ao encontrado neste estudo e preocupante, visto que estes pacientes podem estar retomando os antigos hábitos de vida, o que pode levar à deficiências nutricionais e reganho de peso.

Quanto ao possível aumento de peso no pósoperatório, enfatizando o período superior há dois anos, fica evidente a necessidade de avaliação nutricional periódica da ingestão alimentar, visando a continuidade do processo de reeducação alimentar ${ }^{8,18}$. Christou et al. 6 avaliando pacientes com mais de 16 anos da operação, mostraram redução do percentual de perda de excesso de peso médio de $77,4 \%$ em 10 anos e $55 \%$ em 16 anos, confirmando a possível recuperação de peso $^{6}$.

Antonini et al. ${ }^{3}$ e Cruz et al. ${ }^{7}$ reafirmam que somente o acompanhamento nutricional adequado 
garante o sucesso da operação, evitando complicações nutricionais e prejuízos na perda de peso, reforçando também a necessidade de atenção constante quanto a reeducação alimentar a longo prazo. Ambos os autores confirmam que operação bariátrica é procedimento eficaz para promover perda ponderal, sua manutenção, melhorar parâmetros bioquímicos e reduzir comorbidades, utilizando-se de monitorização nutricional especializada.

Outro aspecto de grande relevância deste estudo, considerando que a maior parte da amostra se faz de pacientes do gênero feminino, é a saúde óssea já que Campos et al. 5 relataram consumo de cálcio insuficiente nos pacientes submetidos há oito anos de bypass em Y-de-Roux; as mulheres apresentaram consumo 50\% menor de cálcio que o recomendado, associado com alta ocorrência de osteopenia.

A atuação do nutricionista no pré e pós-operatório no procedimento cirúrgico bariátrico é importante para preparar e auxiliar o paciente a perder peso de forma saudável e sustentável, para atuar na correção de carências nutricionais e para melhorar a qualidade de vida dos pacientes. Muitas complicações do estado nutricional podem ocorrer após a operação; elas podem ser evitadas com acompanhado periódico.

Cabe a toda equipe multidisciplinar enfatizar a importância do acompanhamento nutricional para que sejam diminuídas complicações metabólicas e recuperação do peso perdido.

\section{CONCLUSÃO}

Verificou-se alta evasão dos pacientes ao acompanhamento nutricional no pós-operatório de cirurgia bariátrica, sendo preocupante a alta desistência principalmente após dois anos da operação. Uma possível alternativa a este problema seria trabalho mais específico no pré-operatório e o uso de diferentes estratégias para individualizar o atendimento nutricional.

\section{REFERÊNCIAS}

1. Aasheim ET, Björkman S, Søvik TT, Engström M, Hanvold SE, Mala T, Olbers T, Bohmer T. Vitamin status after bariatric surgery: a randomized study of gastric bypass and duodenal switch. Am. J. Clin. Nutr. 2009;90(1):15-22.

2. Alvarado R, Alami RS, Hsu G, Safadi BY, Sanchez BR, Morton JM, Curet MJ. The Impact of Preoperative Weight Loss in Patients Undergoing Laparoscopic Roux-en-Y Gastric Bypass. Obes. Surg. 2005; 15: 1282-1286
3. Antonini $D R$, Pereira $C R V$, Simões $N$ et al. Avaliação nutricional de pacientes submetidos à cirurgia bariátrica. Bol. Cirur. Obes. 2001; 2(4): 3.

4. Balduf LM, Kohn GP, Galanko JA, Farrell TM. The Impact of Socioeconomic Factors on Patient Preparation for Bariatric Surgery. Obes. Surg. 2009; 19: 1089-10959.

5. Campos CD, Dalcanale L, Pajecki D, Garrido A, Halpern A. Calcium Intake and Metabolic Bone Disease after Eight Years of Roux-en-Y Gastric Bypass. Obes. Surg. 2008; 18: 386-390.

6. Christou NV, Sampalis JS, Liberman M, et al. Surgery decreases long-term mortality, morbidity, and health care use in morbidly obese patients. Ann. Surg. 2004;240:416-23.

7. Cruz MRR, Morimoto IMI. Intervenção nutricional no tratamento cirúrgico da obesidade mórbida: resultados de um protocolo diferenciado. Rev. Nutr. 2004; 17(2): 263-72.

8. Dalcanale L, Oliveira CPMS, Faintuch J, Nogueira MA, Rondó $P_{1}$ Lima VMR, Mendonça S, Pajecki D, Mancini M, Carrilho FJ. LongTerm Nutritional Outcome After Gastric Bypass. Obes. Surg. 2010, 20:181-187

9. Davies DJ, Baxter JM, Baxter JN. Nutritional deficiencies after bariatric surgery. Obes. Surg. 2007;17(9):1150-8.

10. Francischi RPP, Pereira LO, Freitas CS, Klopfer M, Santos RC, Vieira $P$ et al. Obesidade: atualização sobre sua etiologia, morbidade e tratamento. Rev. Nutr. 2000;13(1):17-28.

11. Hernández MAR, Sancho EM, Fuster MA, Gómez JD, Viñuela IC. Comparación a 5 años de dos técnicas de cirugía bariátrica en pacientes con obesidad mórbida seguidos en consulta enfermera. Rev. Nutr. Hosp. 2009; 24(6):667-675.

12.Instituto Brasileiro de Geografia e Estatística - IBGE. Pesquisa de orçamentos familiares: 2008-2009: Antropometria e Estado Nutricional de Crianças, Adolescentes e Adultos no Brasil. Rio de Janeiro: IBGE; 2010.

13.Júnior WS, Amaral JL, Nonino-Borges CB. Factors Related to Weight Loss up to 4 Years after Bariatric Surgery. Obes. Surg. 2011.

14. Koffman BM, Greenfield J, Ali MI, Pirzada NA. Nurologic Complications After Surgery For Obesity. Muscle Nerve 2006; 33:166-76.

15. Lima LP, Sampaio HAC. Caracterização socioeconômica, antropométrica e alimentar de obesos graves . Rev. Ciência Saúde Coletiva. 2007; 12 (4):1011-1020.

16. Ministério da Saúde. Total de cirurgias de redução de estômago sobe 542\%. (09 de Mar de 2009) [citado em 29 de março de 2011]. Disponível em: http://portal.saude.gov.br/portal/aplicacoes/ reportagensEspeciais/default.cfm?pg=dspDetalhes\&id_ area $=124 \&$ CO NOTICIA $=10008$

17. Padwal R, Klarenbach S, Wiebe N, Hazel M, Birch D, Karmali S, et al. Bariatric Surgery: A Systematic Review of the Clinical and Economic Evidence. J. Gen. Intern. Med. 2011; 26(10):1183-94.

18. Pedrosa IV, Burgos MGPA, Souza NC, Morais CN. Aspectos nutricionais em obesos antes e após a cirurgia bariátrica. Rev. Col. Bras. Cir. 2009; 36(4): 316-322.

19. Poirier $P$, Cornier M, Mazzone T, Stiles $S$, Cummings $S$, Klein $S$, McCullough P, Fielding CR, Franklin BA. Bariatric Surgery and Cardiovascular Risk Factors: A Scientific Statement From the American Heart Association. J. Am. Heart Assoc.; 2011;123:1683-1701.

20. Segal A, Fandiño J. Indicações e contra-indicações para realização das operações bariátricas. Rev. Bras. Psiquiatr. 2002, 24(Suppl 3):68-72.

21. Shankar P, Boylan M, Sriram K. Micronutrient deficiencies after bariatric surgery. Nutrition. 2010; 26(11-12):1031-7.

22. Toledo CC, Camilo GB, Guimarães RL, Moraes FR, Júnior CS. Qualidade de Vida no Pós-Operatório Tardio de pacientes submetidos à Cirurgia Bariátrica. Rev. APS. 2010;13(2):202-9. 